\title{
Control en cascada en la automatización del proceso de elaboración de pegamento implementando un PAC System RX3i y una HMI
}

\author{
Cascade control in the automation of the glue making process by implementing a PAC System \\ RX3i and an HMI \\ E. Flores-García ${ }^{a}$, J.C. Quezada-Quezada ${ }^{b}$, R.V.H. Calderón-Medina ${ }^{c}$, A.S. Hernández-López ${ }^{d}$
}

\begin{abstract}
:
Cascade control is a commonly employed control technique in automated industrial processes due to the advantages it offers. This control strategy involves the controller of the primary variable, called the master or outer controller, and the controller of the secondary variable, called the slave or inner controller; the most important consideration in developing a cascade control system is that inner or secondary control must be faster than outer one. According to the complexity level of processes, these are usually structured in different stages through which the control variable may be exposed to disturbances that affect its behavior and control, which is where cascade control is focused. The present work constitutes a terminal academic project as implementation, focused on industry process automation, in which the application of a cascade control is proposed as a solution alternative for temperature control in the manufacturing process of glue SBC-55, by integrating automation technology such as a PAC System RX3i, SCADA, and an HMI.
\end{abstract}

\section{Keywords:}

Cascade control, Control loop, HMI, PAC System, SCADA

\section{Resumen:}

El control en cascada es una técnica de control comúnmente empleada y de gran utilidad en procesos industriales automatizados, debido a las ventajas que ofrece. En esta estrategia de control interviene el controlador de la variable principal denominado controlador maestro o externo, y el controlador de la variable secundaria denominado esclavo o interno; la consideración más importante para desarrollar un sistema de control en cascada es que el control interno o secundario debe ser más rápido que el externo. De acuerdo con el grado de complejidad de los procesos, éstos suelen estar estructurados en distintas etapas, a través de las cuales la variable de control puede estar expuesta a perturbaciones que afectan su comportamiento y su control, que es hacia donde se orienta el control en cascada. El presente trabajo constituye un proyecto académico terminal de implementación, con enfoque a la automatización de procesos en la industria, en el cual se propone la aplicación de un control en cascada como alternativa de solución para el control de temperatura en el proceso de elaboración de pegamento SBC-55, integrando tecnología de automatización tal como un PAC System RX3i, SCADA, y una HMI.

\section{Palabras Clave:}

Control en cascada, HMI, Lazo de control, PAC System, SCADA

\section{Introducción}

El presente trabajo comprende la integración de tecnologías PAC-HMI y SCADA, principalmente, para la automatización de un proceso de tipo industrial, como parte de la aplicación del conocimiento adquirido por los estudiantes durante su formación profesional en Ingeniería en Tecnologías de Automatización, a través del desarrollo de un proyecto terminal.

\footnotetext{
a Autor de correspondencia, Universidad Autónoma del Estado de Hidalgo, Escuela Superior de Tizayuca, https://orcid.org/0000-00024606-8217, email: efloresg@uaeh.edu.mx

b Universidad Autónoma del Estado de Hidalgo, https://orcid.org/0000-0003-3125-3708, email: jcarlos@uaeh.edu.mx

c Universidad Autónoma del Estado de Hidalgo, https://orcid.org/0000-0002-7292-6051, email: rafael_calderon@uaeh.edu.mx d Universidad Autónoma del Estado de Hidalgo, https://orcid.org/0000-0003-1903-5142, email: he346893@uaeh.edu.mx
} 
El proceso de elaboración de pegamento, en este caso $\mathrm{SBC}-55$, requiere de un control de temperatura preciso que permita asegurar la calidad del producto final, que se realiza a través de la mezcla de diferentes componentes; y en la empresa a la que está enfocado el proyecto, actualmente este proceso se lleva a cabo de forma manual por un operador. Por lo que, con la propuesta planteada en este trabajo, se pretende volver más eficiente el proceso y garantizar el cumplimiento de los estándares de calidad deseados en el producto final; mediante la implementación de un control automático en cascada a través de dos lazos de control de tipo PID.

Control en casada. Esta estructura consiste en dos lazos; un interno y un externo. El lazo interno se enfoca en garantizar que el sistema sea estable mediante la regulación de variables clave para el control del sistema, a través de un set point (SP) que proporciona el lazo externo, teniendo como objetivo cumplir con un rendimiento óptimo. [1]

SCADA. El proceso funcional de los sistemas SCADA (Supervisory Control And Data Acquisition) abarca desde la recopilación de los datos, hasta la toma de acciones de control en un proceso o planta, llevando los datos a la unidad de control para ser interpretados y posteriormente procesados para proveer la información mostrada en la pantalla de monitoreo, de tal manera que en el momento en que el operador elija una acción de control sobre el proceso se realice la interacción hombre-máquina en campo y en la planta. [2]

PAC System. El RX3i pertenece a la familia de Controladores de Automatización Programables, PACs. Posee un único procesador de control y un entorno universal de programación para posibilitar la transportabilidad de la aplicación a través de múltiples plataformas hardware. Es una tecnología industrial orientada al control automatizado avanzado, al diseño de equipos para laboratorios y a la medición de magnitudes analógicas. [3]

HMI (Human-Machine Interface). Es un conjunto de hardware y software usado por un operador $u$ otros usuarios para la interacción directa con el sistema de control y monitoreo del proceso automatizado, a través de un panel de control y/o pantalla maestra, de tipo gráfico y virtual [4]; en este trabajo se implementa mediante el QuickPanel integrado en el PAC, así como en la computadora personal donde se desarrolla la HMI.

Los esquemas de control en cascada han sido utilizados durante más de 60 años, para mejorar el rendimiento de los lazos cerrados de control, tomando como motivo principal la compensación del efecto de las perturbaciones sobre el sistema, así como las demoras o retardos; actualmente este tipo de control es utilizado para la regulación de variables típicas como el flujo, la presión, o la temperatura. [1]

La consideración más importante para desarrollar un sistema de control en cascada es que el control interno o secundario debe ser más rápido que el externo. Cuando se aplica correctamente esta técnica de control, se obtienen resultados altamente satisfactorios ya que se logra que todo el circuito sea más estable y con un tiempo de respuesta menor. [5]

En la actualidad existen industrias que poseen procesos de alta complejidad, por lo que es necesario que se dividan en etapas, y éstas a su vez sean controladas con una mejor y más precisa estrategia de control.

\section{Planteamiento del problema}

Se tiene un sistema donde el control de temperatura depende del control de nivel y temperatura del jacket, y a su vez el control de nivel depende del control de la temperatura. Los sistemas de control PID en cascada son aplicables en procesos de elevada complejidad y con altos estándares de precisión; sin embargo, se vuelven inviables para medianas y pequeñas empresas en donde la automatización se encuentra en un nivel bajo. Por lo anterior, surge un problema al intentar controlar todo un proceso en donde las variables dependen cíclicamente unas de otras, y lograr un control más preciso; el presente trabajo plantea responder a dicha problemática, proponiendo una alternativa de solución usando la estrategia de control en cascada. [6]

Objetivo General. Implementar un Control en Cascada de dos lazos mediante un PAC System y monitoreo a través de $\mathrm{HMI}$, para mejorar el control en el proceso de elaboración de pegamento SBC-55.

\section{Objetivos específicos:}

- Generar los algoritmos de control en lenguaje Diagrama Escalera mediante el software Proficy Machine Edition.

- Desarrollar una Interfaz Humano-Maquina empleando el software Cimplicity Workbench.

- Utilizar el PAC System RX3i de GE Fanuc para el control en cascada.

- Implementar un sistema SCADA para realizar de manera remota el monitoreo y control del proceso.

- Integrar sensores de nivel y de temperatura RTD PT100.

\section{Desarrollo de la propuesta}

El software Cimplicity ${ }^{\circledR}$ es escalable desde una Interfaz Humano-Máquina (HMI) a un sistema de Control Supervisado y Adquisición de Datos (SCADA) completamente en red. Las capacidades de red inherentes a todos los niveles dentro de la línea de 
productos le permiten alcanzar niveles de integración que prácticamente eliminan la configuración redundante dentro de una red. Se basa en una arquitectura de servidor-cliente que consiste en Servidores y Visores. Los servidores son responsables de la recopilación y distribución de datos. Los espectadores se conectan a los servidores y tienen acceso completo a los datos recopilados para ver y controlar las acciones. [7]

La Tabla 1 muestra la variable de entrada del algoritmo de control, la cual corresponde a una señal física analógica proporcionada por un sensor (\%IA57) RTD tipo Pt100 que mide la temperatura; esta variable está asociada al PAC con dirección IP 10.0.0.10.

\begin{tabular}{lccc}
\hline Variable & Dirección & Descripción & IP \\
\hline \multirow{2}{*}{ SENSOR } & $\%$ AI57 & Medición del RTD & 10.0.0.10 \\
& & & \\
\hline & Tabla 1. Variables del PAC.
\end{tabular}

Para generar la base de datos, se instalaron los software SQL Server 2014 y Management studio; Proficy Machine Edition y Cimplicity. Se creó una base de datos mediante SQL Server 2014 Management studio.

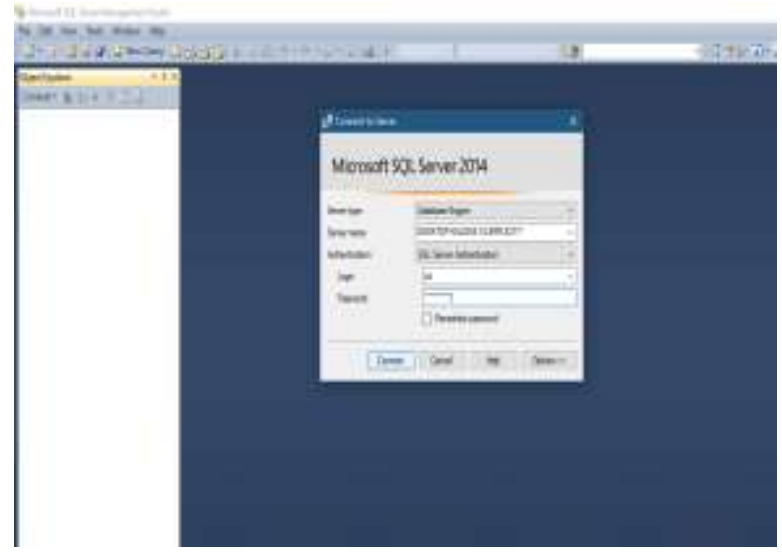

Imagen 1. Conexión con servidor DESKTOP/CIMPLICITY.

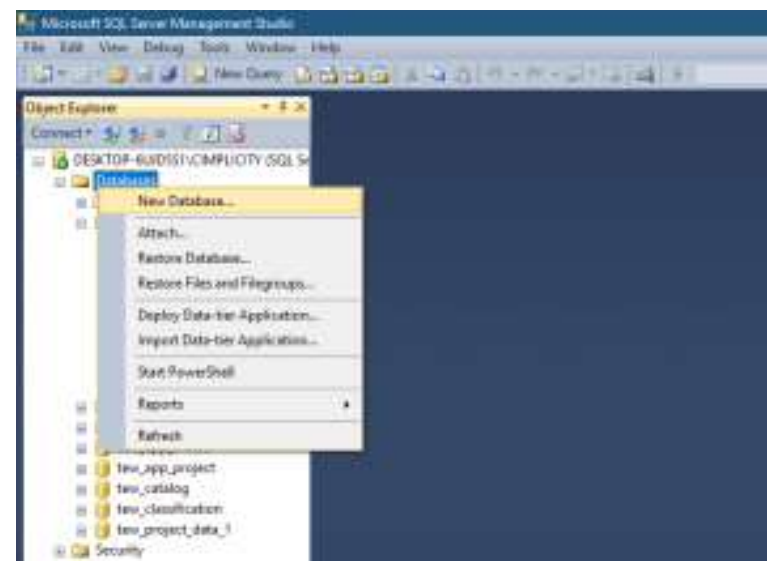

Imagen 2. Creación de una nueva base de datos.

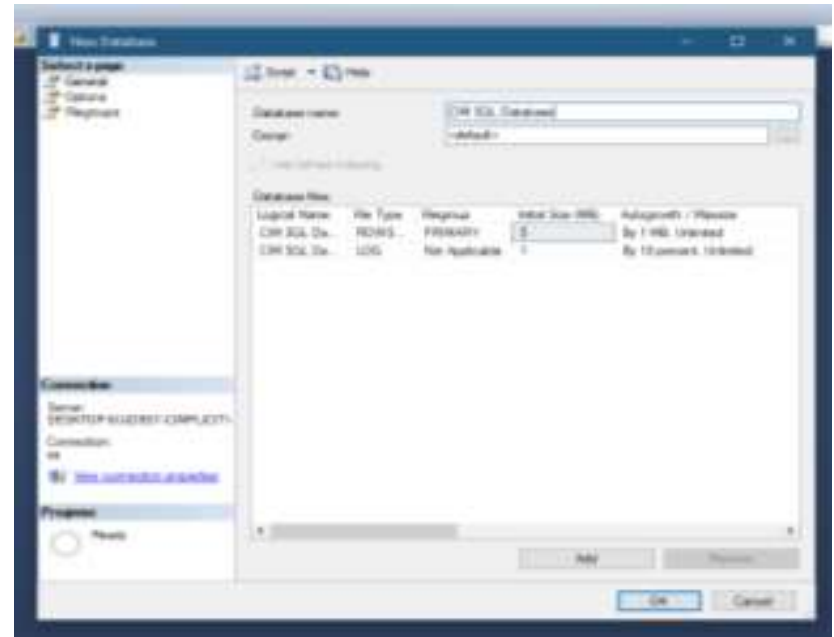

Imagen 3. Nombre de la base de datos.

La configuración del servidor se realiza eligiendo el ícono de Windows y buscando el programa $O D B C$ DATA SOURCE.

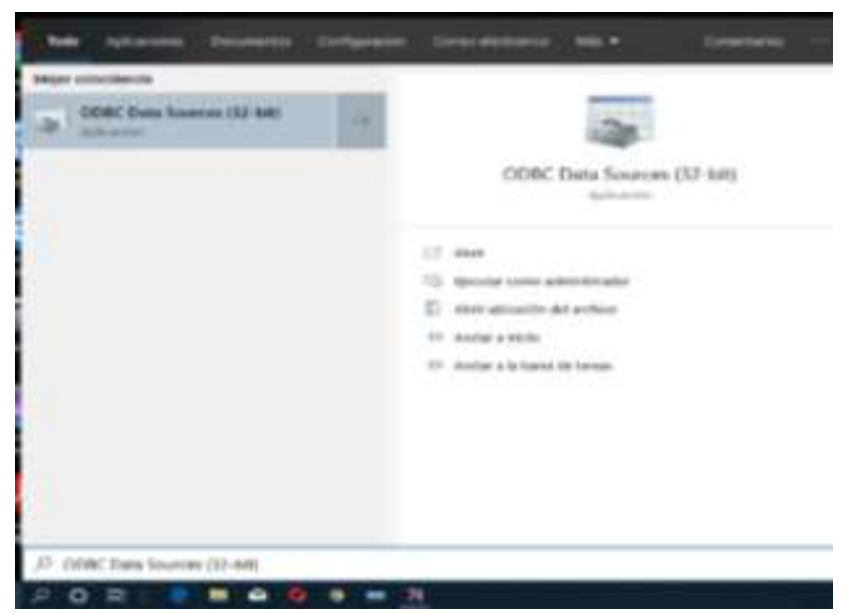

Imagen 4. Búsqueda del programa $O D B C$ en Windows.

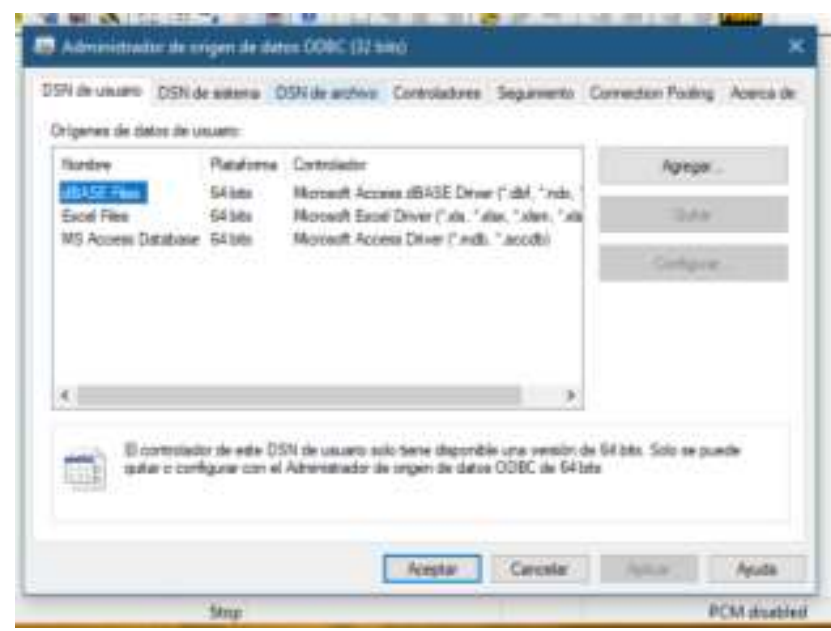

Imagen 5. Interfaz de ODBC DATA SOURCE.

Para la creación del servidor, en DSN se selecciona el sistema ejecutado. 


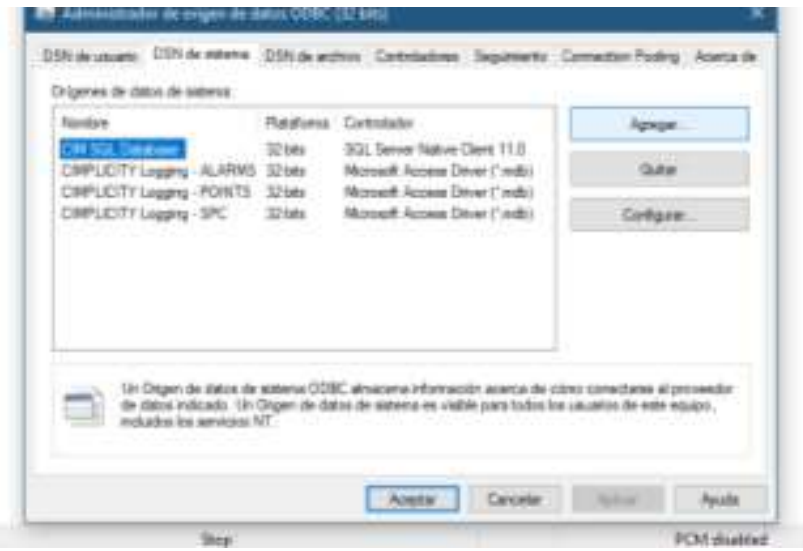

Imagen 6. DSN de sistema.

Se agrega la opción de SQL Server Native Client 11.0.

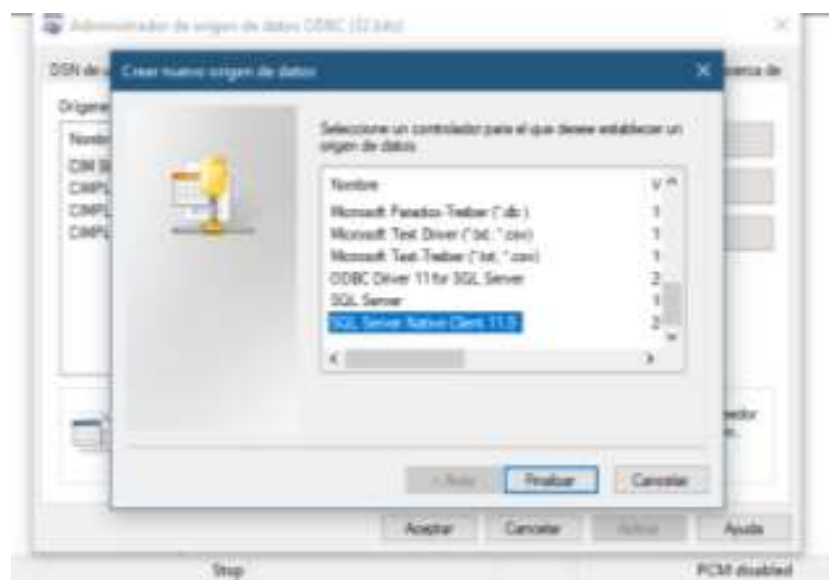

Imagen 7. Creación de un nuevo origen de datos.

Como muestra la Imagen 8, se requiere especificar el nombre del servidor, y en el apartado Server seleccionar el "nombre de tu equipo/CIMPLICITY".

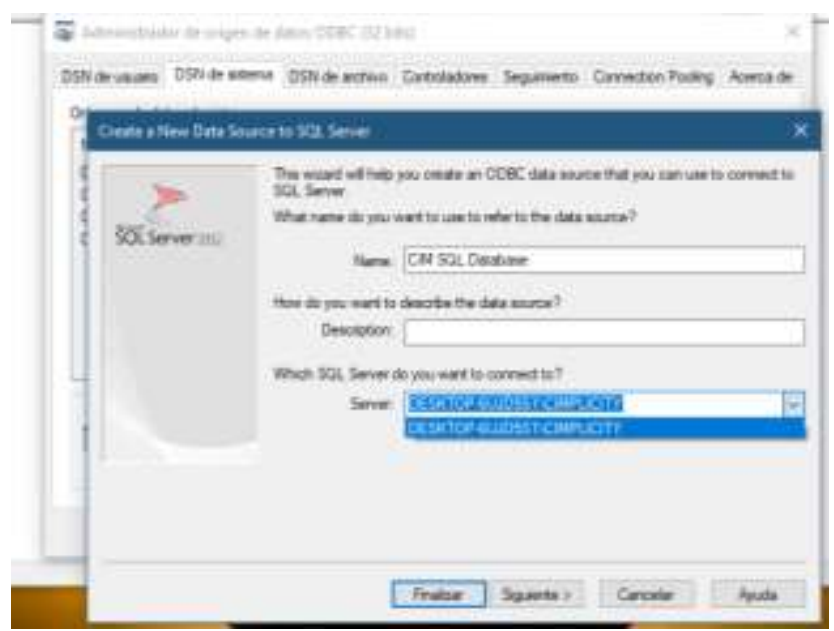

Imagen 8. Selección del servidor a conectar.

Ingresar los datos de usuario y contraseña que fueron creados en la instalación del software SQL Server 2014.

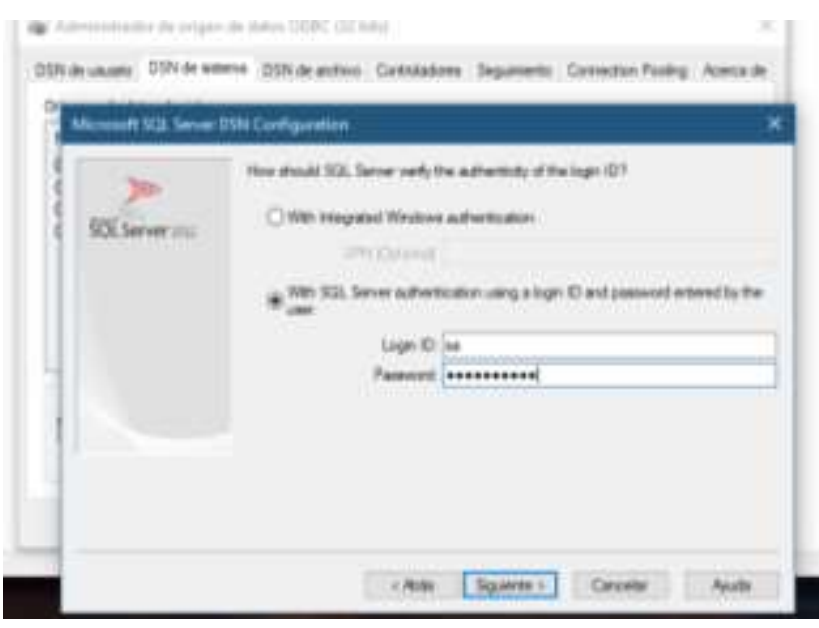

Imagen 9. Ingreso de usuario y contraseña.

Se selecciona la base de datos que fue creada en el software "Management studio".

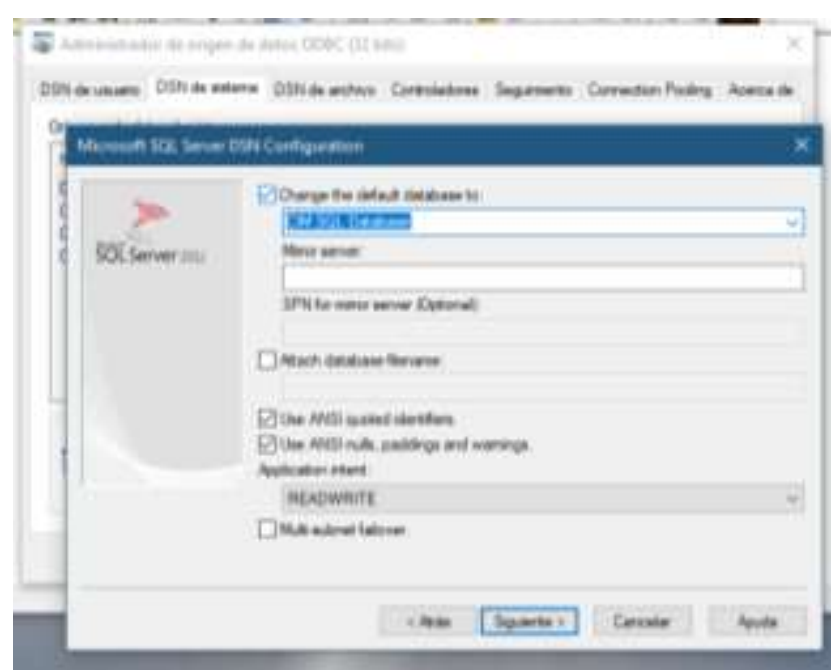

Imagen 10. Selección de la base de datos.

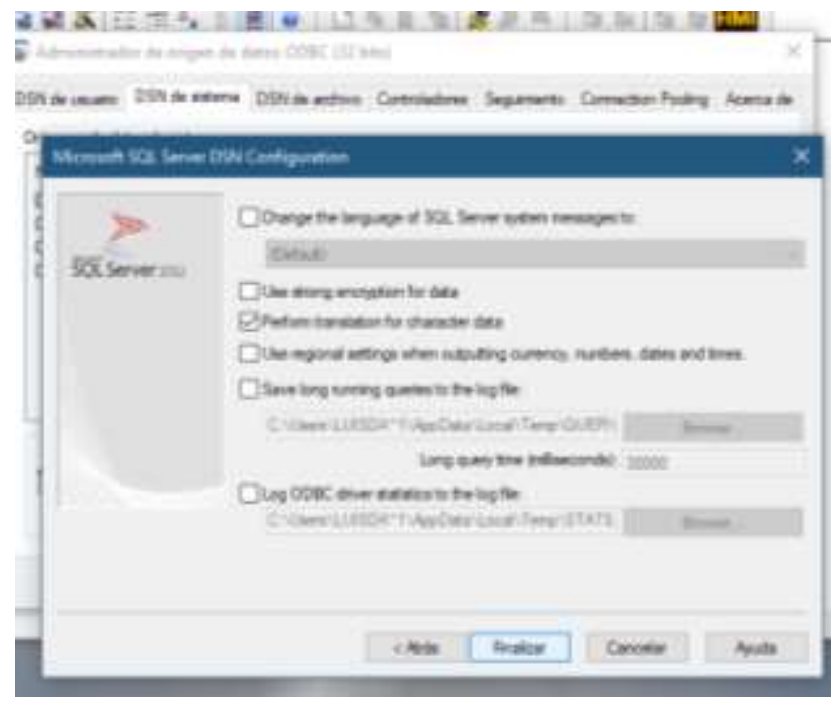

Imagen 11. Finalización de la configuración del servidor 
La configuración del PAC se realiza a través del software Proficy Machine Edition, en este caso para efectuar su interconexión con el software Cimplicity.

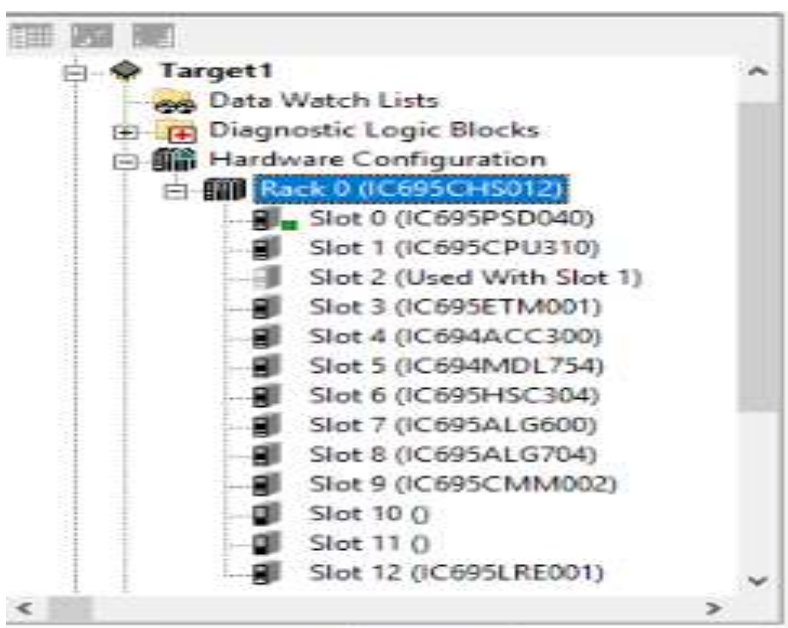

Imagen 12. Configuración del hardware del PAC.

\begin{tabular}{|c|c|}
\hline Description & \\
\hline \multicolumn{2}{|l|}{ Documentation Address } \\
\hline Family & PACSystems $F \times 3 i$ \\
\hline Controler Target Name & ventladoe 1 \\
\hline Update Rate [ms] & 250 \\
\hline Swwep Time [ms] & attine \\
\hline Controler Status & Otfine \\
\hline Scheduling Mode & Normal \\
\hline Force Compact PVT & Trus \\
\hline Enable Shared Varisbles & False \\
\hline Process System Enabled & False \\
\hline DLB Hearibeat [ms] & 1000 \\
\hline Enhanced Security & False \\
\hline Compression Level & Normal \\
\hline Physical Port & ETHERINET \\
\hline IP Address & 10.00 .11 \\
\hline \multicolumn{2}{|l|}{ تladdrional Configuation } \\
\hline spector & \\
\hline
\end{tabular}

Imagen 13. Direccionamiento del target 1 para el PAC.

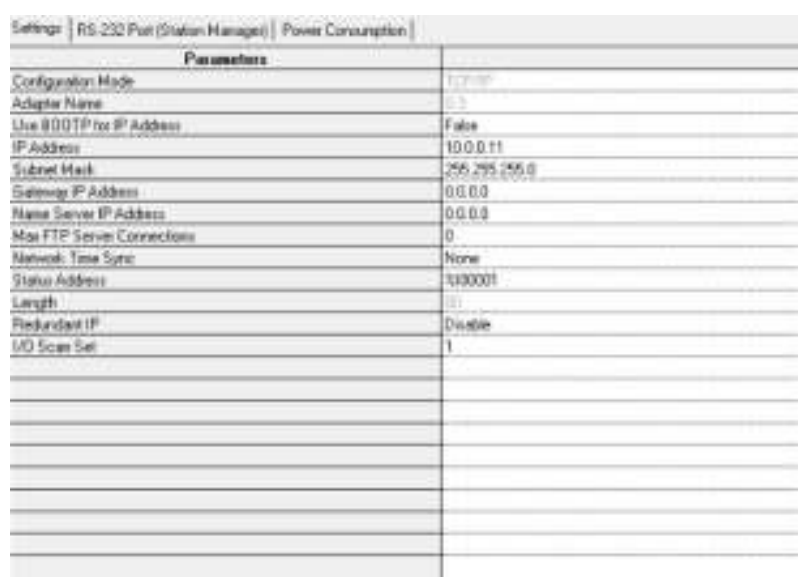

Imagen 14. Configuración del módulo de comunicación del PAC.

La Imagen 15 muestra la configuración del módulo de entradas analógicas IC695ALG600, donde se ha seleccionado el instrumento de medición a utilizar.

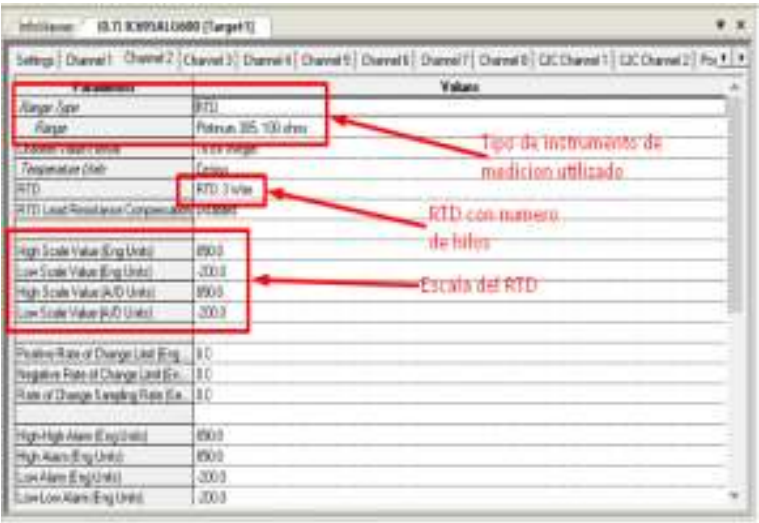

Imagen 15. Configuración del módulo para la señal de entrada analógica proporcionada por el RTD.

Para la programación en Cimplicity y la configuración de la base de datos, se abre un proyecto nuevo; se ejecuta una ventana de creación donde, en el apartado de protocolos, se selecciona la opción "S90 TRIPLEX', y en el de opciones, "Database Logger : point" y "Database Logger : A\&E \& App", y se asigna el nombre y la carpeta de destino, como se muestra en la Imagen 16.

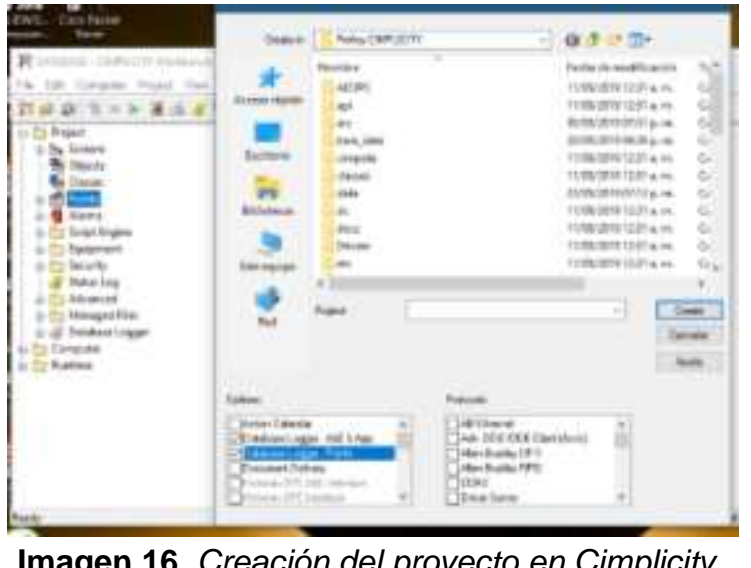

Posteriormente, las ventanas de configuración irán apareciendo de acuerdo con la aceptación de los términos, como muestra la Imagen 17.

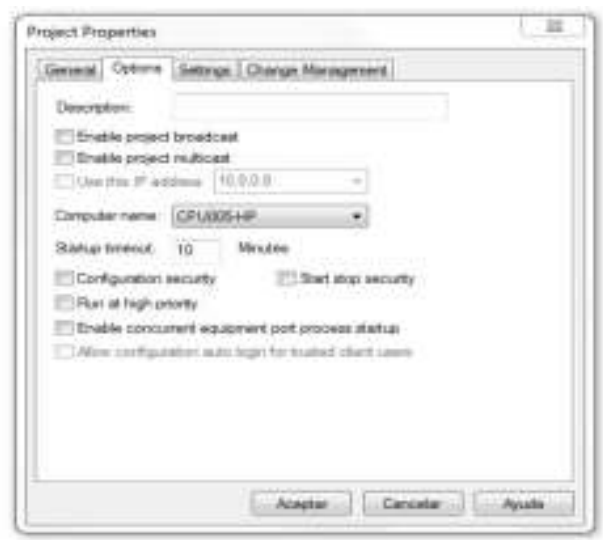

Imagen 17. Propiedades del proyecto. 
En el apartado Project Wizard, se genera la comunicación que se establecerá entre la computadora y el PAC, agregando las direcciones IP; Imagen 18.

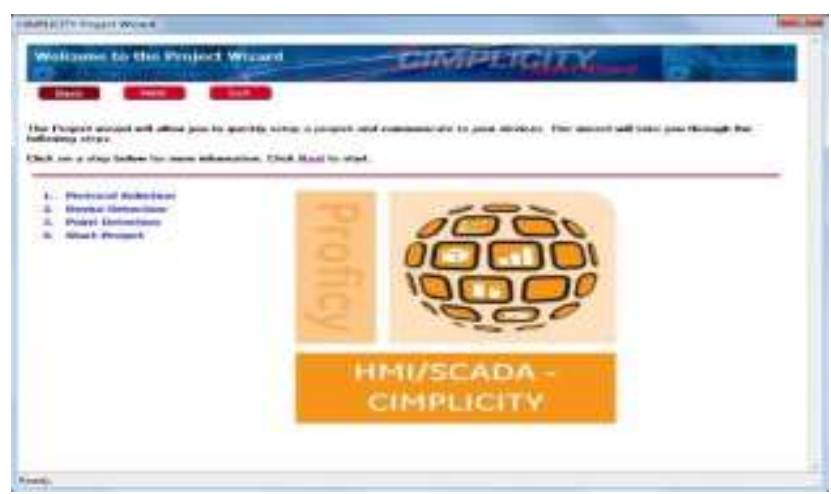

Imagen 18. Project Wizard para la comunicación.

Se selecciona S90 TRIPLEX en el apartado siguiente; Imagen 19.

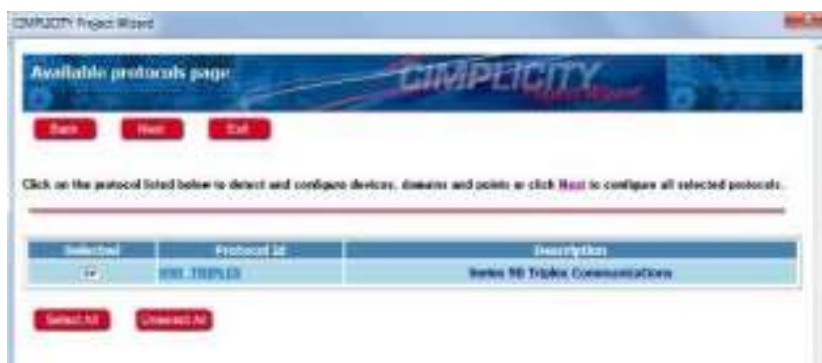

Imagen 19. Selección de Protocol ID.

Una vez creado el New Project Wizard, se genera un nuevo punto, asignándole un nombre asegurando que cuente con la misma dirección IP que el PAC, como se observa en la Imagen 20 y 21.

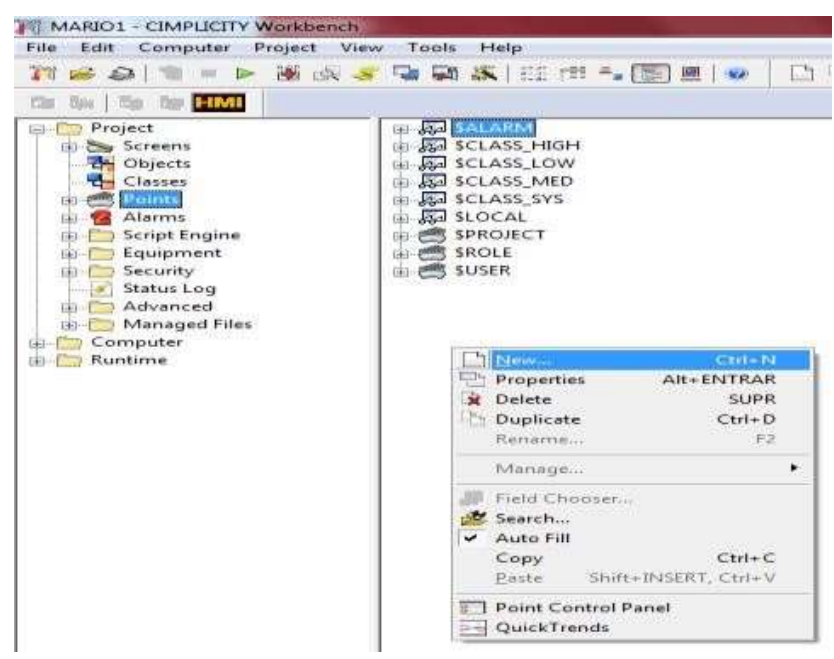

Imagen 20. Creación de un nuevo punto.

Se asigna la dirección al punto como muestra la Imagen 22
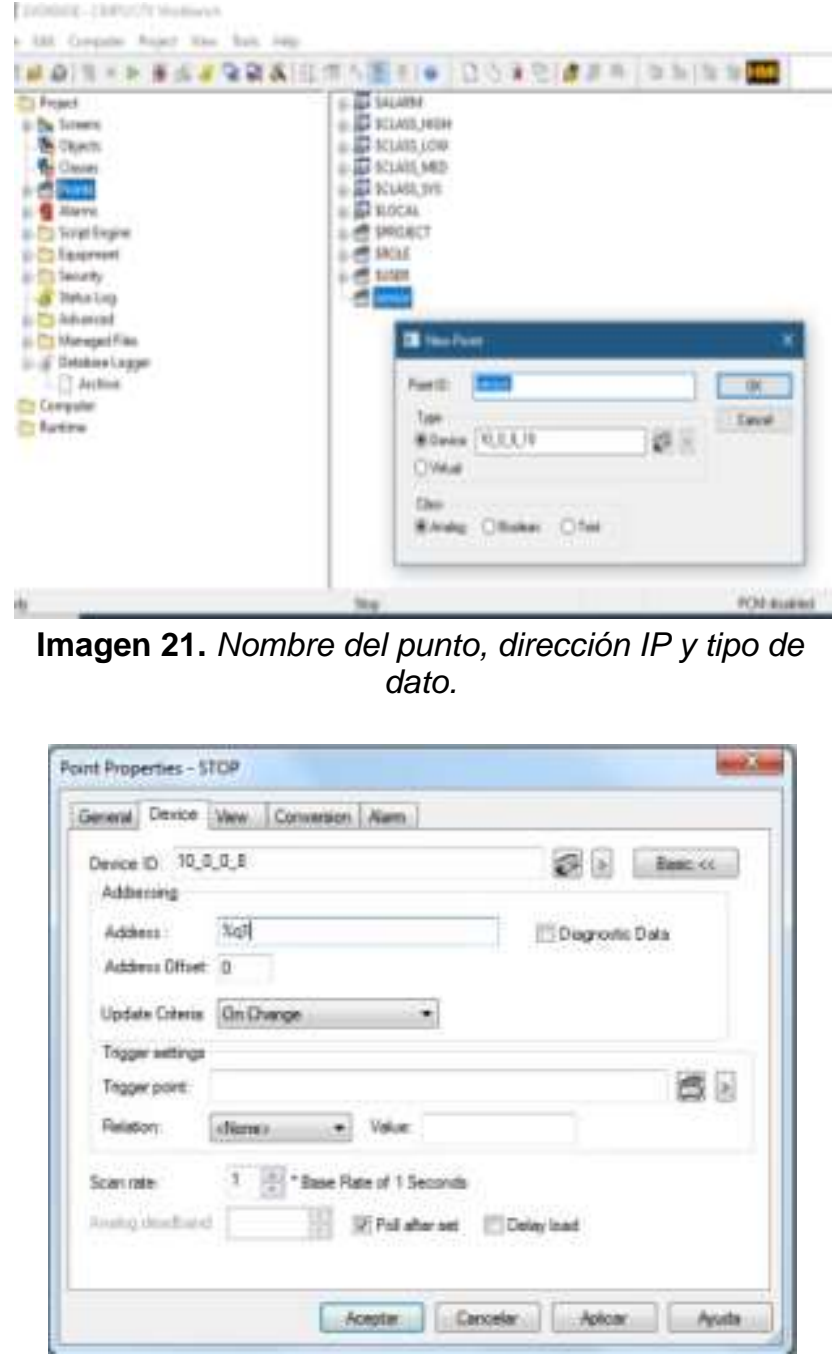

Imagen 22. Point Address.

Habiendo creado los puntos necesarios, se diseña un nuevo Screen y se inicia el proyecto.

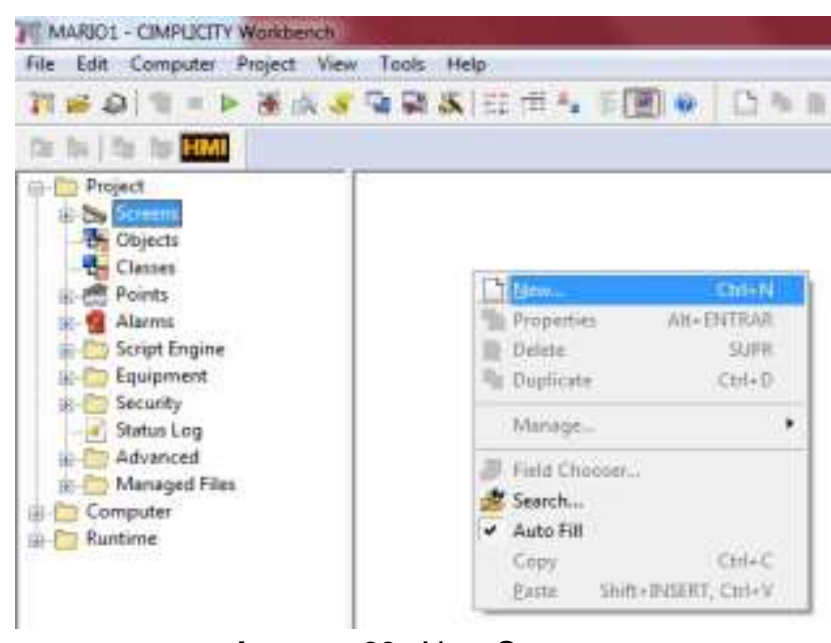

Imagen 23. New Screen

Se configura la conexión de la base de datos con el software Cimplicity para poder iniciar la comunicación. 


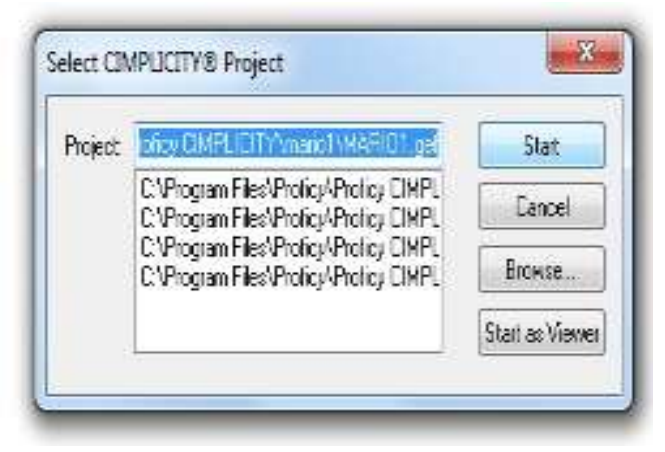

Imagen 24. Botón de START.

Se ejecuta "Database Logger", donde se encuentran las tablas que se han configurado para la conexión de la base de datos con el servidor.

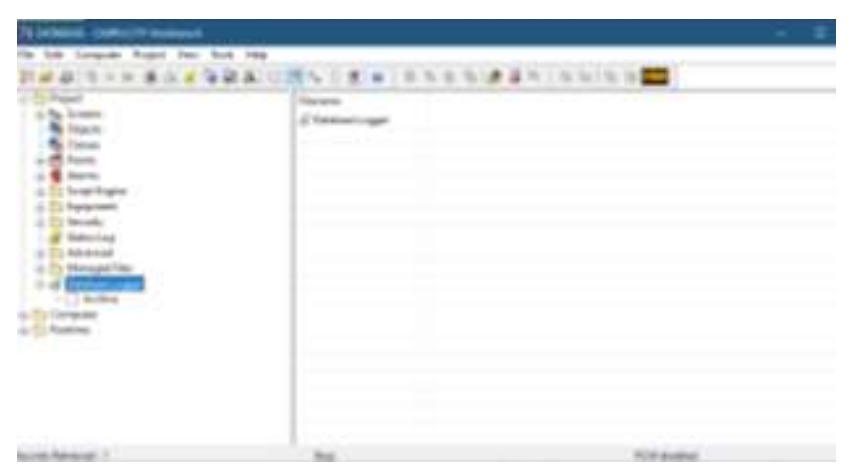

Imagen 25. Database logger.

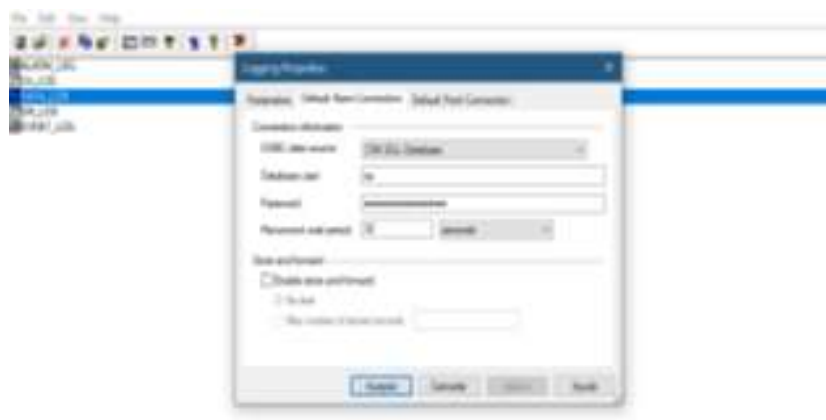

Imagen 26. Configuración de la tabla "datalogger" para la conexión con el servidor de alarma.

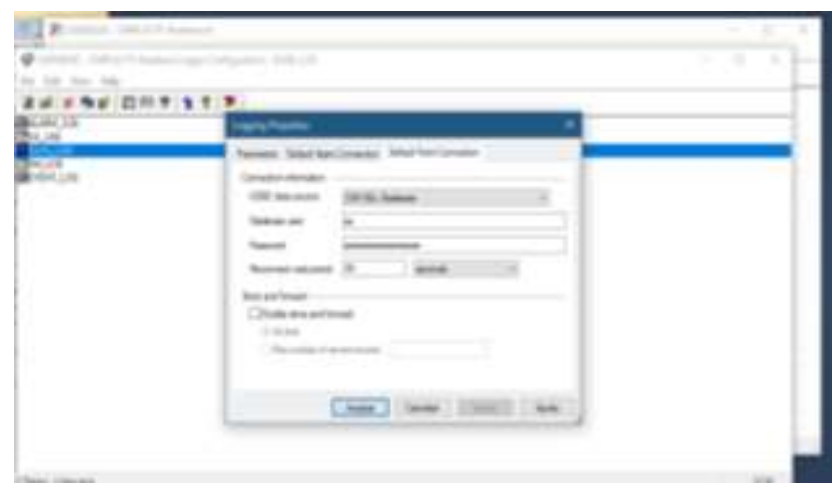

Imagen 27. Configuración de la tabla "datalogger" para la conexión con el servidor de puntos.
Se debe seleccionar el DSN que fue configurado anteriormente para la conexión con las tablas que aparecen en Cimplicity.

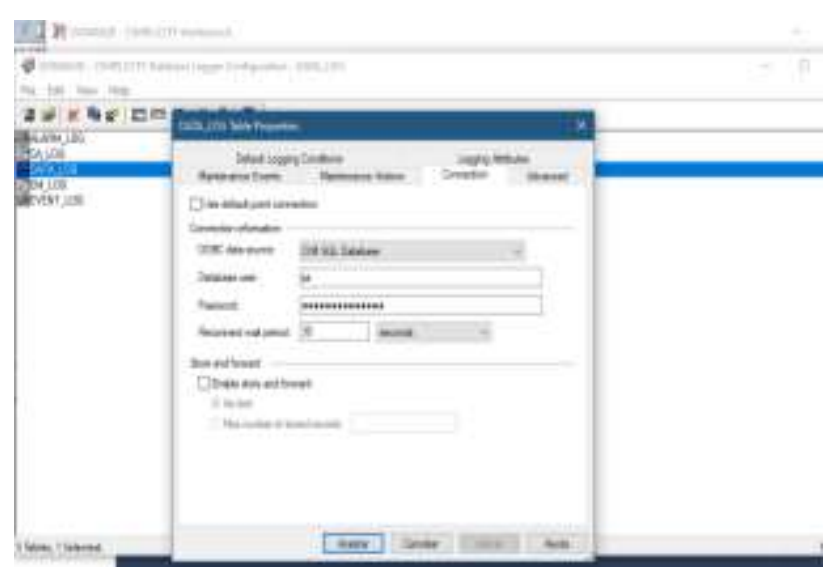

Imagen 28. Configuración de la tabla "datalogger" para la conexión con el servidor con la tabla de propiedades.

Se crea el punto o tag que estará enviando los datos que registra el punto para la base de datos.
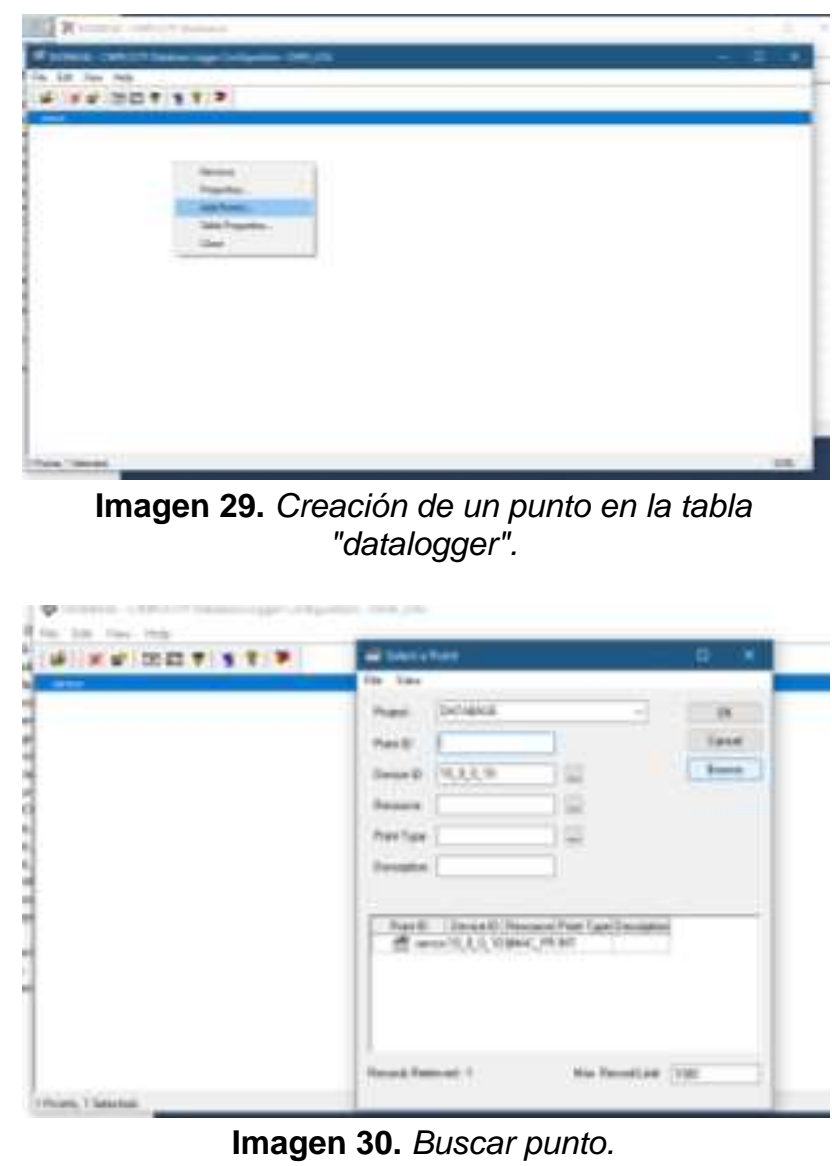

En la Imagen 31-34, se presenta cómo verificar los valores en los puntos que fueron asignados, para que se guarden los valores que se generan en la base de datos. También se observan las tablas que fueron configuradas en el software Cimplicity. 


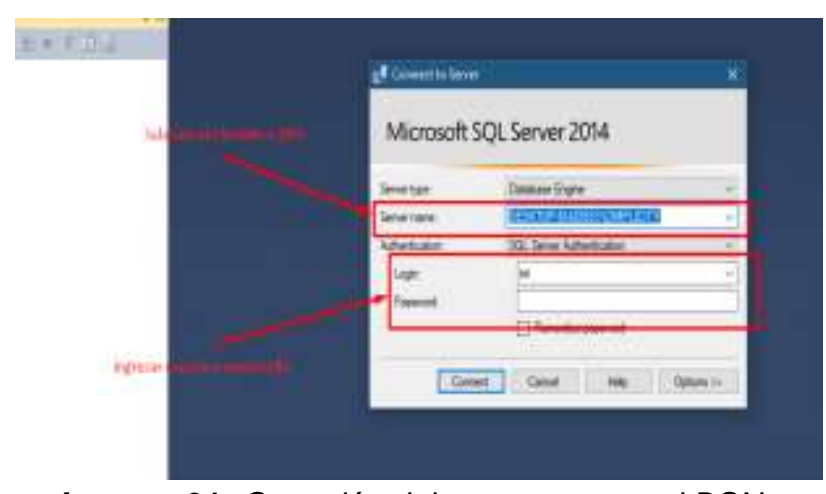

Imagen 31. Conexión del programa con el DSN.

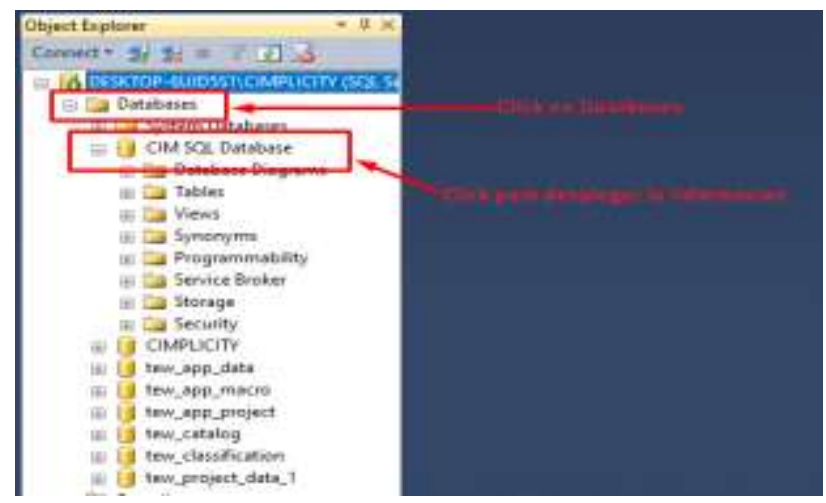

Imagen 32. Selección de la base de datos.

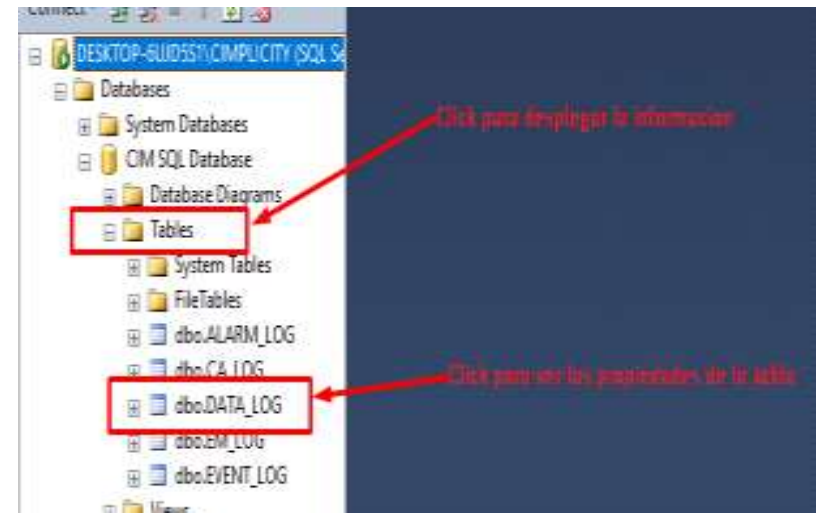

Imagen 33. Visualización de las tablas configuradas con el software Cimplicity.

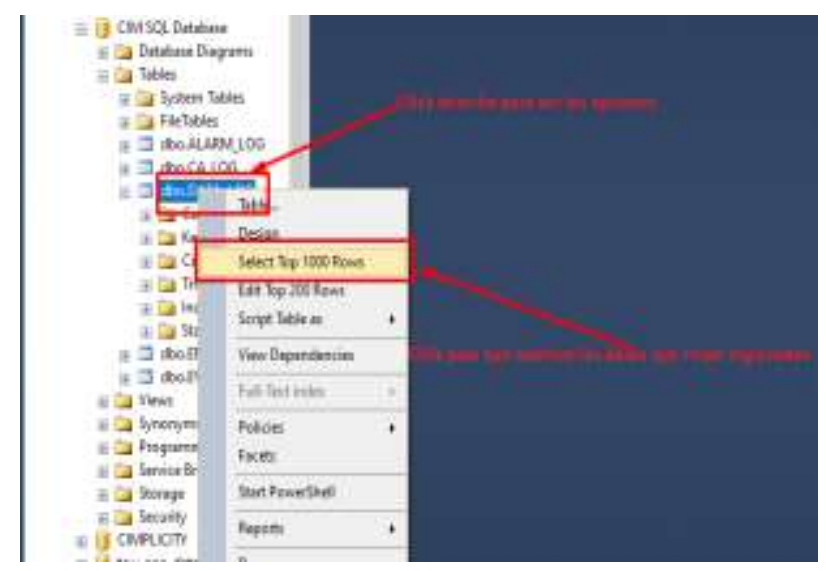

Imagen 34. Selección para mostrar los últimos 1000 datos que fueron registrados de cada punto.
El sistema propuesto implica dos $\mathrm{HMl}$; una interfaz principal para la selección del modo de visualización del control, y otra para la simulación del proceso. Se contemplan cuatro algoritmos de control en diagrama escalera; uno principal, uno para cada subsistema del control en cascada, y otro para las condiciones del sistema.

La Tabla 2 muestra la variable del algoritmo de control principal; \#ALLOW_ON (\%S7) es una señal del sistema del PAC y significa siempre energizado; las subrutinas de funcionamiento se encuentran bajo esta condición.

\begin{tabular}{ccc}
\hline Variable & Direccionamiento & Descripción \\
\hline \#ALLOW_ON & $\%$ S007 & Siempre energizada
\end{tabular}

Tabla 2. Variable en el algoritmo de control principal.

La Imagen 35 muestra el algoritmo de control principal, el cual se compone de tres subrutinas. CONDITIONS siempre permanece en estado activo, ya que la variable \#ALLOW_ON es la que la energiza; esta subrutina es para el escalamiento de la variable de proceso (PV, por sus siglas en inglés de Process Variable), y para el punto de ajuste (SP, por sus siglas en inglés de Set Point) o temperatura deseada del sistema, de ambos lazos de control. La subrutina MASTER es para el control del lazo maestro; y SLAVE para el control del lazo esclavo.

La Tabla 3 muestra las variables en la HMI principal del sistema a controlar. La Imagen 36 presenta a la $\mathrm{HMI}$ principal para el sistema de control de temperatura, tal como se propone la conexión de los elementos en el sistema modelo donde se realizan las pruebas. Las HMI, ejecutándose en una computadora personal, se comunican mediante protocolo Ethernet con el PAC, el cual recibe y envía señales de tipo discreto y analógico correspondientes a las variables de campo.

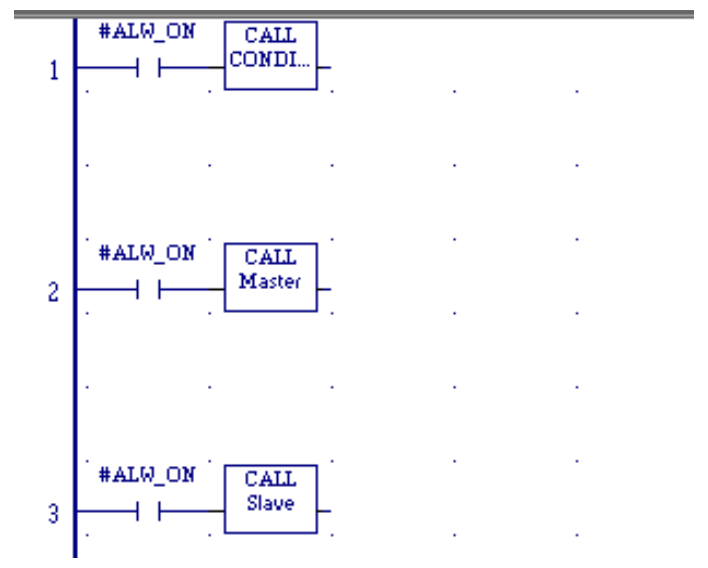

Imagen 35. Algoritmo de control principal del sistema mediante el software Proficy Machine Edition. 


\begin{tabular}{cc}
\hline $\begin{array}{c}\text { Variable en la } \\
\text { HMI }\end{array}$ & Direccionamiento \\
\hline Physical HS & $\begin{array}{c}\text { Alarma virtual del estado de paro de } \\
\text { emergencia real HS_F (\%181) }\end{array}$ \\
\hline $\begin{array}{c}\text { Electrical } \\
\text { safeguards }\end{array}$ & $\begin{array}{c}\text { Alarma virtual del estado de las } \\
\text { protecciones del sistema PROTECTION } \\
(\% / 82)\end{array}$ \\
\hline HS virtual & $\begin{array}{c}\text { Paro de emergencia virtual (paro por } \\
\text { software), controla el estado de HS_V } \\
\text { (\%M4) }\end{array}$ \\
\hline PV1 & $\begin{array}{c}\text { Variable del proceso a controlar, está } \\
\text { dada por el sensor de temperatura } \\
\text { (\%AI57) }\end{array}$ \\
\hline
\end{tabular}

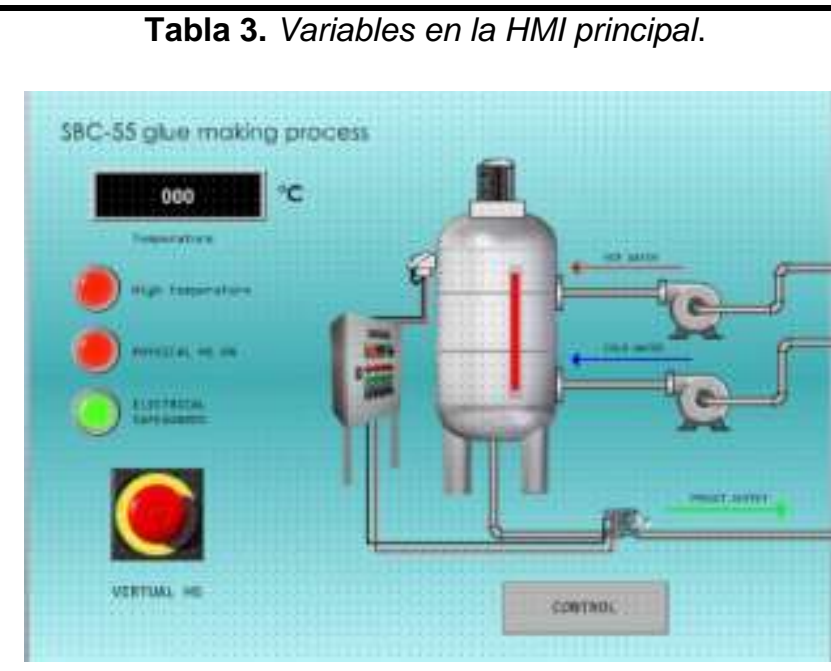

Imagen 36. HMI Principal.

\section{Escalamiento de señales SP y PV a la variable de temperatura}

Para las señales de SP y PV se realizó un escalamiento para la variable de temperatura en un intervalo de 25 a $100^{\circ} \mathrm{C}$. EI SP se direcciona hacia un registro de memoria y la PV hacia una entrada analógica; ambas son de tipo entero a 16 bits, por lo que su intervalo de trabajo en formato decimal es de $32768\left(2^{15}\right)$. La variable SP se manipula desde la HMI para el control de temperatura. Para la variable PV se utiliza una señal estándar de control de 4-20 mA. Ambas señales se condicionan a un valor máximo de 30600 , que es dividido en la HMI para obtener el valor máximo de temperatura. La Tabla 4 muestra el escalamiento respectivo para la PV y SP.

La Imagen 37 muestra el algoritmo de control para el escalamiento de la PV; el valor de la señal analógica PV1 (\%AI57), correspondiente a la temperatura real del sistema, se mueve de forma continua al registro auxiliar (\%R1), el cual corresponde a la variable que se divide en la HMI para mostrar esa temperatura real en un intervalo de 25 a $100^{\circ} \mathrm{C}$. Los bloques LE INT y GE INT se usan para limitar la PV, de manera que no exceda el límite inferior de 0 y el límite superior de 30600.
Las reglas lógicas de los algoritmos de acondicionamiento son:

- Si PV1 es menor o igual que cero, entonces AUX_1 $(\% \mathrm{M} 10)=1$ y la variable de registro auxiliar $(\% \mathrm{R} 1)=0$.

- Si PV1 es mayor o igual que 30600, entonces AUX_2 $(\% \mathrm{M} 11)=1$ y la variable de registro auxiliar $(\% \mathrm{R} 1)=30600$.

Estas condiciones son aplicadas de la misma manera para la variable PV2 (\%AI59).

\begin{tabular}{cccc}
\hline Decimal & SP $\left({ }^{\circ} \mathbf{C}\right)$ & PV (mA) & PV $\left({ }^{\circ} \mathbf{C}\right)$ \\
\hline $\mathbf{0}$ & 25 & 4 & 25 \\
\hline $\mathbf{1 6 2 5 7}$ & 53.125 & 10 & 53.125 \\
\hline $\mathbf{3 0 6 0 0}$ & 100 & 20 & 100 \\
\hline
\end{tabular}

Tabla 4. Escalamiento de $S P$ y $P V$.

La ecuación utilizada para realizar el escalamiento es:

$$
P V(\text { units })=\left(\frac{I-4}{16}\right) *\left(P V_{\text {Max }}-P V_{\text {Min }}\right)+P V_{\text {Min }} \text { Ec. } 1
$$

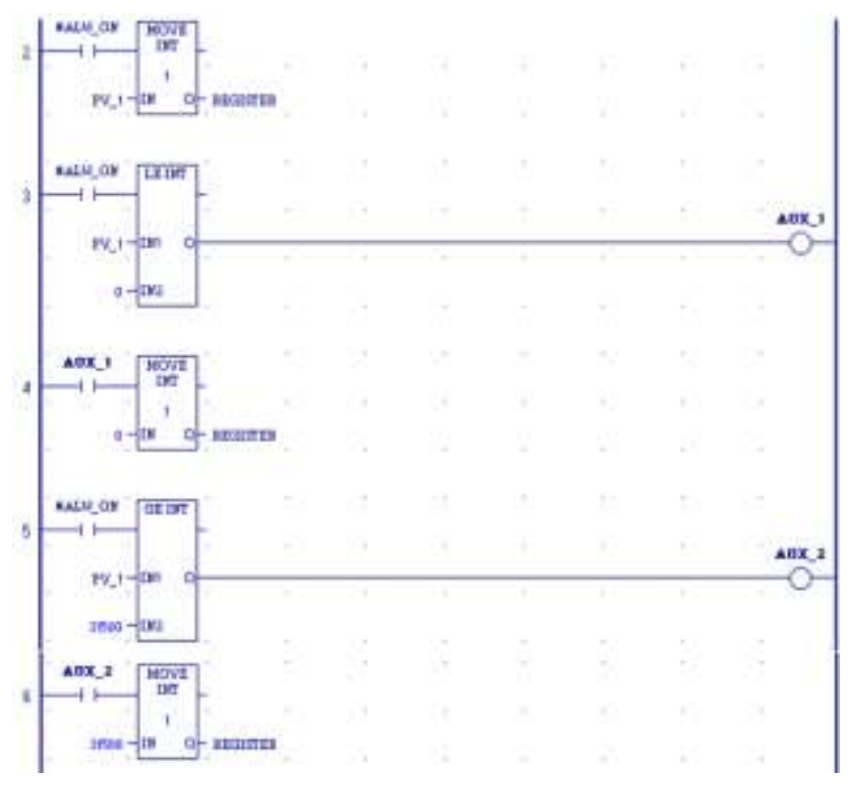

Imagen 37. Escalamiento de PV1.

\section{Lógica de control en lazo cerrado propuesta}

La configuración de control en lazo cerrado se utiliza en procesos continuos que usan la variable de proceso, PV, como retroalimentación para el control automático. Las Imágenes 39 y 40 muestran la propuesta de algoritmo para ambos lazos de control en cascada de temperatura en un reactor. El bloque BLK CLR WORD se propone para limpiar los 40 registros de memoria de 16 bits que utiliza el bloque PID IND; el control maestro inicia en el registro \%R50, y el esclavo en el \%R100; por lo tanto, el registro es la entrada del bloque BKL CLR WORD. 
Los bloques BLK MOV INT se utilizan para asignar los parámetros de ajuste y sintonización del controlador. La variable SP (\%R10) se manipula desde la $\mathrm{HMl}$; la variable PV (\%AI57) proviene del sensor de temperatura (RTD Pt100) como señal analógica de retroalimentación; el contacto AUT_MAN se emplea para seleccionar el modo de operación del controlador PID; y las variables para aumentar y disminuir la variable de control (CV, por sus siglas en inglés de Control Variable) son para el control de la variable en modo de operación manual.

La Tabla 5 muestra las variables utilizadas para el control en lazo cerrado del lazo maestro; esas variables sólo son manipulables en el modo de operación manual del controlador PID. El modo automático/manual del controlador PID se establece a través de un selector virtual desde la $\mathrm{HMI}$, y está direccionado hacia la variable AUT_MAN (\%M16) en el algoritmo. La HMI muestra una ventana auxiliar para la sintonización del PID, es decir, para la asignación del valor de las constantes $\mathrm{Kp}$, Ki y $\mathrm{Kd}$, y a los registros de referencia del controlador, \#referencia $+5 ;+7 ;+6$; respectivamente. Las variables del controlador esclavo $\mathrm{CV}$ se encuentran dependientes del lazo maestro y por lo tanto no son manipulables en la HMI.

\begin{tabular}{|c|c|}
\hline $\begin{array}{c}\text { Variable en la } \\
\text { HMI }\end{array}$ & Descripción \\
\hline $\begin{array}{c}\text { Botón subir } \\
\text { CV }\end{array}$ & Incremento de la variable CV \\
\hline $\begin{array}{c}\text { Botón bajar } \\
\text { CV }\end{array}$ & Decremento de la variable CV \\
\hline Kp & $\begin{array}{c}\text { Asigna un valor a la ganancia } \\
\text { proporcional }\end{array}$ \\
\hline $\mathbf{K i}$ & Asigna un valor a la ganancia integral \\
\hline Kd & $\begin{array}{c}\text { Asigna un valor a la ganancia } \\
\text { derivativa }\end{array}$ \\
\hline
\end{tabular}

Tabla 5. Variables de lazo cerrado en la HMI.

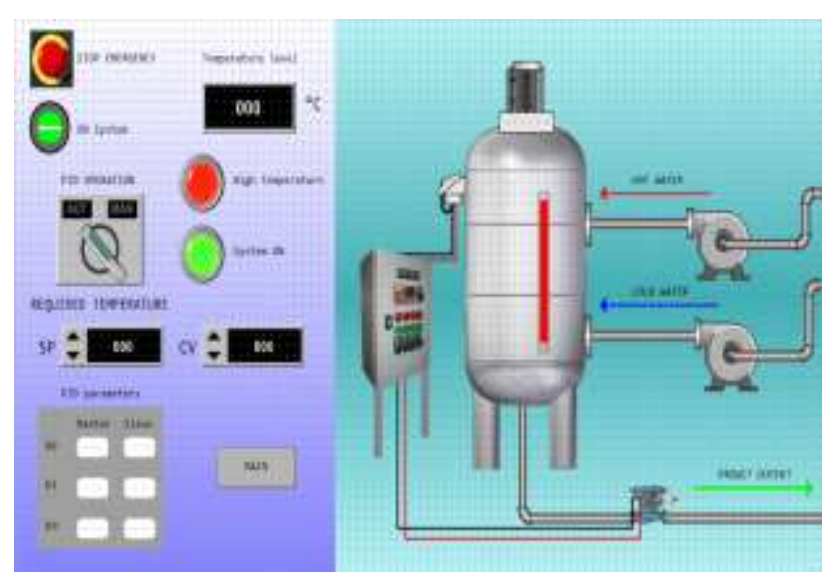

Imagen 38. HMI de control.
La Imagen 39 e Imagen 40 muestran, respectivamente, el algoritmo del lazo de control maestro y esclavo.

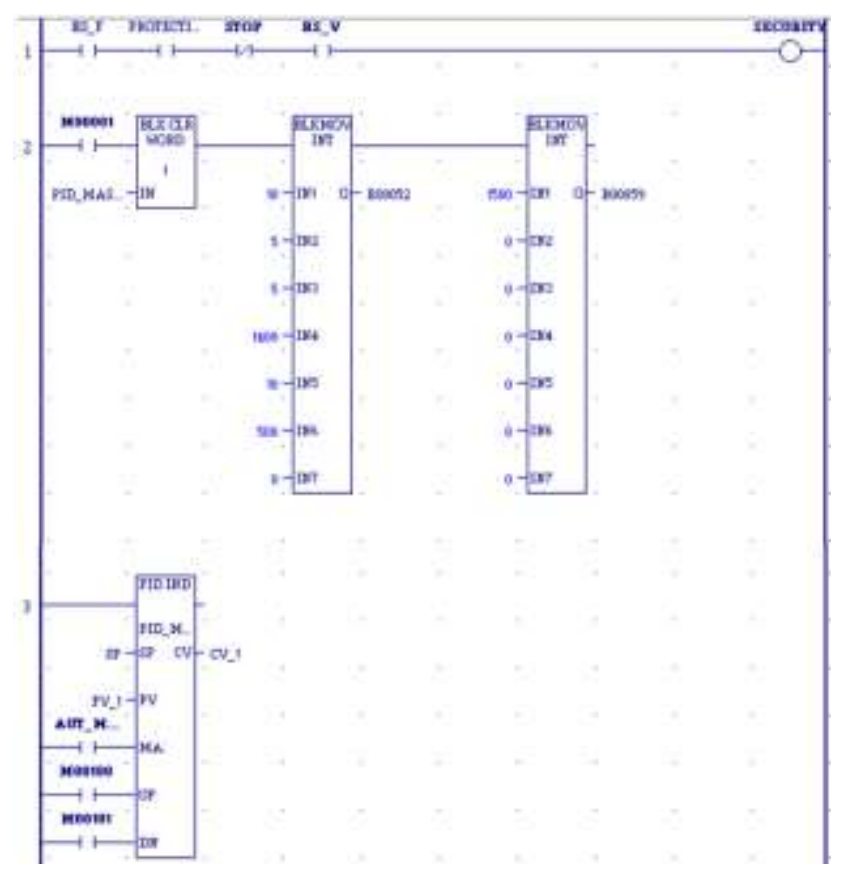

Imagen 39. Lazo de control maestro.

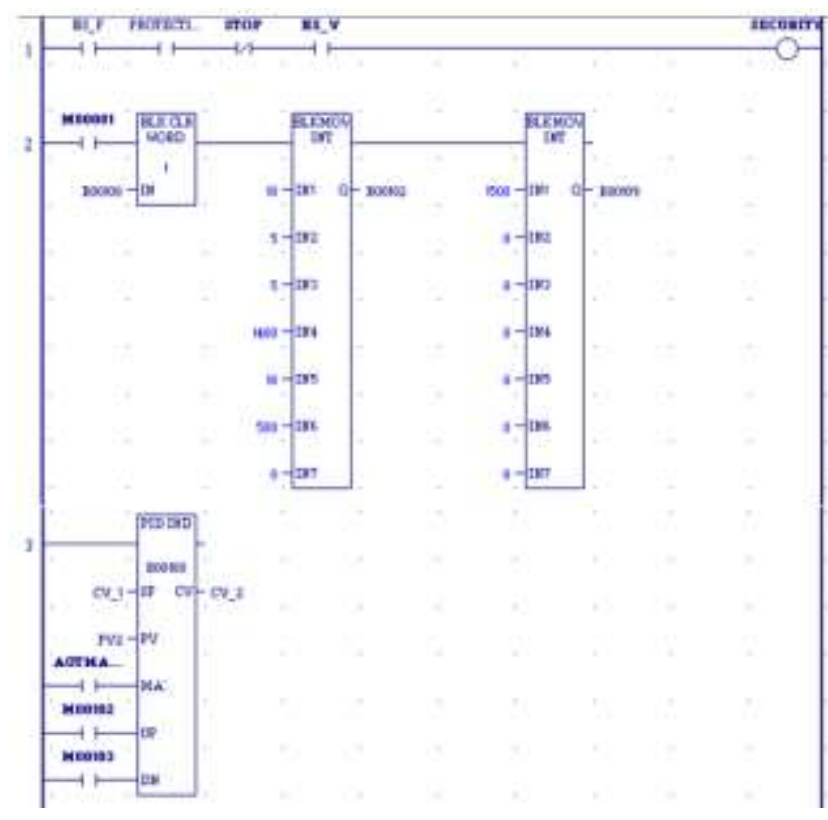

Imagen 40. Lazo de control esclavo.

\section{Conclusiones}

El presente trabajo constituye un proyecto académico terminal de aplicación práctica e implementación, con enfoque a la automatización de procesos productivos, empleando tecnología de tipo industrial, como propuesta de alternativa de solución a la problemática que se presenta en el desarrollo de los procesos. El objetivo principal es la implementación de un control en cascada 
de dos lazos en el proceso de elaboración de pegamento SBC-55, a través de un PAC System RX3i y monitoreo mediante una HMI diseñada en software para SCADA. En el desarrollo de las HMI se propuso incluir la mayor parte de programación, debido a que esto permite que el tiempo de barrido o scan de la lógica de control sea más eficiente. Asimismo, las reglas de control, monitoreo y animación han de generar un entorno amigable y seguro con los operadores del sistema, respetando lo establecido en la norma ANSI/ISA-101.012015. Los algoritmos de control propuestos fueron realizados en diagrama escalera, considerado el lenguaje de mayor popularidad a nivel industrial. La lógica de control en el PAC System contiene el interlocking de seguridad del proceso para garantizar el paro total del sistema en caso de falla eléctrica, sobrecalentamiento, falta de comunicación entre la computadora donde se encuentran las HMl y el PAC. La asignación y el ajuste de los valores de las ganancias del controlador PID se realizó de manera empírica, mediante procedimiento de prueba y error. En la presente propuesta se ha planteado un modo de control temperatura-temperatura que pretende mejorar la seguridad y el comportamiento del proceso en sus reactores químicos, conociendo permanentemente el estado del sistema a través de la base de datos generada, que es fundamental para la toma de decisiones en el proceso de producción.

\section{Referencias}

[1] M. Zárate, J. García y S. Calderón, Cascade control of a class of chemical reactors based on entropy production shaping, London: Springer, 2017.

[2] J. L. Ochoa, M. B. Valenzuela, F. M. Cirett y R. A. Luque, «Un modelo para desarrollar sistemas tipo SCADA en entornos productivos,» Universidad de Sonora, Sonora, 2018.

[3] Ge Fanuc Automation, «PACSystems Rx3i,» Mayo 2005. [En línea] Available: https://www.cimtecautomation.com/parts/c-356-emersonrx3i-pac-systems.aspx. [Último acceso: 22 Febrero 2020].

[4] National Instruments, «National Instruments,» 9 Julio 2015. [En línea]. Available: https://www.ni.com/es-mx.html. [Último acceso: 16 Febrero 2020].

[5] C. Smith y C. Armando, Control Automático de procesos: Teoría y Práctica, México: Limusa S.A de C.V, 1991.

[6] J. G. Álvarez, J. E. Moreno y E. F. Ramírez, «Diseño e implementación de un sistema de control cascada en la planta de intercambio térmico,» Informador técnico, 2017.

[7] GE Fanuc, «ASTOR,» 19 Julio 2001. [En línea]. Available: https://www.astor.com.pl/wsparcie/dokumentacjatechniczna/pobierz/4680. [Último acceso: 06 Marzo 2020]. 